\title{
AM-FM MODELS, PARTIAL HILBERT TRANSFORM, AND THE MONOGENIC SIGNAL
}

\author{
Chuong T. Nguyen and Joseph P. Havlicek \\ School of Electrical and Computer Engineering \\ University of Oklahoma, Norman, OK 73019 USA
}

\begin{abstract}
We propose a new algorithm to compute the frequency modulation functions associated with the well-known monogenic signal. The new algorithm extracts the frequency modulation functions without requiring an auxiliary process to estimate local orientation. In addition, we show that, in situations where a multi-scale multiorientation decomposition is required to analyze a signal, the partial Hilbert transform approach computes AM-FM functions similar to those obtained by the monogenic signal while maintaining a more efficient signal representation.
\end{abstract}

Index Terms - AM-FM, monogenic signal, partial Hilbert transform

\section{INTRODUCTION}

Fourier analysis is an important tool for analyzing and representing a stationary signal as a sum of pure sinusoids having constant amplitudes and constant frequencies. However, in practice many important signals are nonstationary in the sense that the amplitude envelope and frequency content are time varying (or space varying). Obvious examples include seismic survey data, radar returns, a variety of communication signals, and many biomedical signals [1]. The AM-FM model

$$
f(\mathbf{x})=a(\mathbf{x}) \cos [\varphi(\mathbf{x})]
$$

has been widely used to represent such signals, where, for the $2 \mathrm{D}$ case we are concerned with in this paper, $a(\mathbf{x}): \mathbb{R}^{2} \rightarrow \mathbb{R}^{+}$is a smoothly varying non-negative amplitude modulation (AM) function and $\varphi(\mathbf{x}): \mathbb{R}^{2} \rightarrow \mathbb{R}$ is the phase modulation function. The frequency modulation (FM) function $\nabla \varphi(\mathbf{x})$ carries a rich local description of the surface pattern orientation and granularity.

The AM-FM model (1) is ill-posed in the sense that infinitely many pairs of $a(\mathbf{x})$ and $\varphi(\mathbf{x})$ exist which satisfy the equality (1). In his seminal 1D paper [2], Gabor used the Hilbert transform (HT) to disambiguate the AM-FM modeling problem by constructing a complex signal extension called the analytic signal. In arbitrary dimensions, any given complex extension associates unique AM and FM functions with a real signal which may be obtained directly by taking the magnitude of the complex signal and by differentiating the argument of the complex signal.

While Gabor's analytic signal is well-defined in $1 \mathrm{D}$, extension to higher dimensions is nontrivial. The main reason is that there is no straightforward means of extending the Hilbert transform into multiple dimensions. Peyrin, Zhu, and Goutte [3] defined a 2D complex signal by performing a 1D Hilbert transform along a specified direction; such transforms are frequently referred to as partial Hilbert

This work was supported in part by the U.S. Army Research Laboratory and the U.S. Army Research Office under grant W911NF-08-1-0293. transform (pHT). Havlicek, Havlicek, and Bovik proposed an adjusted multiplier [4] to enforce harmonic correspondence [5] of the multidimensional pHT and used it to define a multidimensional analytic image [6]. Other related developments include Hahn's single orthant complex signal [7] and the hypercomplex signal of Bülow and Sommer [8], both of which are important but of limited interest for our purposes here because they do not provide a complete representation of all possible signal orientations. Felsberg and Sommer [9] introduced the multidimensional monogenic signal where the 1D HT is replaced by an $n \mathrm{D}$ Riesz transform. Independently, Larkin et al. [10] used the same signal model to study fingerprints.

While other approaches exist for computing AM-FM models without an explicit complex extension such as the Teager-Kaiser energy operator [11] and the quasi-local approximation [12,13], the explicit complex extension approaches including the $\mathrm{pHT}$ model and the monogenic signal have remained highly popular. These two models have been successfully applied in many practical applications such as motion estimation [14], fingerprint modeling [10], and texture analysis [15] just to name a few. Both compute an explicit complex extension for the real signal by adding an imaginary part that is equal to the pHT for the partial Hilbert approach and equal to the Riesz transform for the monogenic signal. The main advantage of the monogenic signal lies in the isotropic kernel of the Riesz transform, which tends to avoid undesirable rippling in the AM function; such amplitude ripples are characteristic of the AM functions typically obtained via the pHT. As will be described in more detail below, the main advantage of the pHT approach is that it enables the FM functions to be obtained directly without the need for an auxiliary orientation estimation procedure as is required with the monogenic signal approach.

In this paper, our attention is focused on the $\mathrm{pHT}$ and monogenic signal approaches. We propose a new alternative algorithm for computing the monogenic signal FM functions that avoids the need for an auxiliary orientation estimation procedure. In addition, we demonstrate that in situations where a multi-scale multi-orientation signal decomposition is required, the $\mathrm{pHT}$ approach can deliver equivalent modulation functions to those obtained with the monogenic signal while maintaining a simpler representation. We argue that the pHT method and monogenic signal are both viable approaches. They are closely related and it is in fact possible to formulate a closed form relationship between the two representations although we will not do so here in the interest of brevity. For signals that admit orientations (e.g., spectral support) orthogonal to the direction of action of the pHT, the pHT approach will always suffer from undesirable amplitude rippling that is not present in the monogenic signal. However, pHT based models are always more efficient than the monogenic signal in the sense of requiring only one imaginary component as opposed to two. For signals that do not admit significant spectral support orthogonal to the pHT direction of action, both approaches typically deliver equivalent but slightly different AM-FM interpreta- 
tions of the signal.

\section{COMPUTATION OF THE AM-FM IMAGE MODEL}

While the pHT and the monogenic signal have both been generalized to higher dimensions, we limit our discussion to $2 \mathrm{D}$ signals throughout the paper in the interest of simplicity. Let $f(\mathbf{x}): \mathbb{R}^{2} \rightarrow \mathbb{R}$ be a real-valued signal. We use the hat "へ," to denote Fourier transform, as in $f(\mathbf{x}) \leftrightarrow \widehat{f}(\boldsymbol{\omega})$.

\subsection{Partial Hilbert Transform Approach}

The 2D pHT [16, p. 49] extends the 1D version by performing a 1D HT in the direction specified by a unit vector $\mathbf{e}$. Let $q_{\mathbf{e}}(\mathbf{x})$ be the pHT of $f(\mathbf{x})$ in the direction $\mathbf{e}$ with Fourier spectrum given by

$$
\widehat{q}_{\mathbf{e}}(\boldsymbol{\omega})=-j \operatorname{sgn}\left(\boldsymbol{\omega}^{T} \mathbf{e}\right) \widehat{f}(\boldsymbol{\omega}),
$$

where the signum function is defined by

$$
\operatorname{sgn}(\tau)=\left\{\begin{array}{rr}
+1, & \tau>0 \\
0, & \tau=0 \\
-1, & \tau<0
\end{array}\right.
$$

and $\boldsymbol{\omega}=\left[\begin{array}{ll}\omega_{1} & \omega_{2}\end{array}\right]^{T}$. The 2D complex signal is constructed exactly as in the 1D case, i.e., $z_{\mathbf{e}}(\mathbf{x})=f(\mathbf{x})+j q_{\mathbf{e}}(\mathbf{x})$. As in the $1 \mathrm{D}$ case, the spectrum of the 2D complex signal admits frequency support on precisely half of the frequency space. With $z_{\mathbf{e}}(\mathbf{x})$ the $2 \mathrm{D}$ complex signal obtained via the $2 \mathrm{D} \mathrm{pHT}$, the AM-FM model (1) is computed according to [6]

$$
\begin{aligned}
a_{\mathbf{e}}(\mathbf{x}) & =\left|z_{\mathbf{e}}(\mathbf{x})\right|, \\
\nabla \varphi_{\mathbf{e}}(\mathbf{x}) & =\operatorname{Im}\left[\frac{\nabla z_{\mathbf{e}}(\mathbf{x})}{z_{\mathbf{e}}(\mathbf{x})}\right],
\end{aligned}
$$

where " $\nabla$ " denotes gradient. Note that (4), (5) provides an AM-FM representation that is exact in the sense of achieving equality in (1).

While the algorithm (4), (5) always produces an AM-FM model that achieves equality in (1), the obtained AM function will generally exhibit undesirable amplitude rippling when the input function $f(\mathbf{x})$ admits nontrivial spectral support at orientations orthogonal to e. These ripples are not intuitive and are generally not consistent with human visual perception. They occur because the pHT kernel is directional and not isotropic. Image features that are normal to $\mathbf{e}$ are not represented in a visually intuitive way, and this is a limitation of the pHT approach.

\subsection{Monogenic Signal}

In the context of signal processing applications, Felsberg and Sommer [9] first proposed the Riesz transform as an appropriate multidimensional extension of the HT for constructing a multidimensional analog of the analytic signal. In 2D, they defined a three component complex signal $z_{\mathrm{mo}}(\mathbf{x})$, viz., the monogenic signal, according to

$$
z_{\mathrm{mo}}(\mathbf{x})=f(\mathbf{x})+i f_{1}(\mathbf{x})+j f_{2}(\mathbf{x}),
$$

where $i$ and $j$ are two distinct orthogonal hypercomplex imaginary units. The functions $f_{1}(\mathbf{x})$ and $f_{2}(\mathbf{x})$ are two components of the Riesz transform with Fourier spectra given by

$$
\begin{aligned}
& \widehat{f}_{1}(\boldsymbol{\omega})=\frac{\omega_{1}}{\|\boldsymbol{\omega}\|^{2}} \widehat{f}(\boldsymbol{\omega}), \\
& \widehat{f}_{2}(\boldsymbol{\omega})=\frac{\omega_{2}}{\|\boldsymbol{\omega}\|^{2}} \widehat{f}(\boldsymbol{\omega}) .
\end{aligned}
$$

In practice, the monogenic signal is computed using an auxiliary local orientation estimate $\phi(\mathbf{x})$ according to

$$
\begin{aligned}
f(\mathbf{x}) & =a_{\mathrm{mo}}(\mathbf{x}) \cos \left[\varphi_{\mathrm{mo}}(\mathbf{x})\right] \\
f_{1}(\mathbf{x}) & =a_{\mathrm{mo}}(\mathbf{x}) \sin \left[\varphi_{\mathrm{mo}}(\mathbf{x})\right] \cos [\phi(\mathbf{x})], \\
f_{2}(\mathbf{x}) & =a_{\mathrm{mo}}(\mathbf{x}) \sin \left[\varphi_{\mathrm{mo}}(\mathbf{x})\right] \sin [\phi(\mathbf{x})]
\end{aligned}
$$

where $a_{\mathrm{mo}}(\mathbf{x})$ is the monogenic $\mathrm{AM}$ function and $\varphi_{\mathrm{mo}}(\mathbf{x})$ is the monogenic local phase. From these relations, the AM and FM functions can be calculated analytically. The AM is given by

$$
a_{\mathrm{mo}}(\mathbf{x})=\sqrt{f^{2}(\mathbf{x})+f_{1}^{2}(\mathbf{x})+f_{2}^{2}(\mathbf{x})} .
$$

and the monogenic local phase is given by

$$
\varphi_{\mathrm{mo}}(\mathbf{x})=\frac{f_{1}(\mathbf{x})}{\left|f_{1}(\mathbf{x})\right|} \arctan \left[\frac{\sqrt{f_{1}^{2}(\mathbf{x})+f_{2}^{2}(\mathbf{x})}}{f(\mathbf{x})}\right] .
$$

Note that the monogenic phase function (10) is wrapped and lies in the interval $[-\pi, \pi]$. Therefore, the FM functions, which are calculated by the gradient of $\varphi_{\mathrm{mo}}(\mathbf{x})$, will be (potentially grossly) inaccurate at locations where jumps of integer multiples of $\pi$ occur due to branch cuts in the arctan function because of phase wrapping. This problem can be circumvented by estimating the local orientation $\phi(\mathbf{x})$ in (8) and then constructing an imaginary image $q_{\mathrm{mo}}(\mathbf{x})$ as proposed in $[10,17]$. The imaginary image $q_{\mathrm{mo}}(\mathbf{x})$ is given by

$$
q_{\mathrm{mo}}(\mathbf{x})=f_{1}(\mathbf{x}) \cos [\phi(\mathbf{x})]+f_{2}(\mathbf{x}) \sin [\phi(\mathbf{x})] .
$$

The FM functions are then computed directly from (5), where $z_{\mathbf{e}}(\mathbf{x})=f(\mathbf{x})+j q_{\mathrm{mo}}(\mathbf{x})$.

As the kernel of the Riesz transform is isotropic, the computed AM in (9) does not exhibit amplitude rippling as is observed with the pHT approach. Nevertheless, the computation of the FM functions in the monogenic approach is more complicated than in the case of the $\mathrm{pHT}$ as the monogenic approach requires a priori knowledge of the local orientation $\phi(\mathbf{x})$.

\section{NEW ALGORITHM FOR COMPUTING THE MONOGENIC FM}

In the complex signal extension framework, the pHT computes the imaginary part of the complex signal as $a_{\mathbf{e}}(\mathbf{x}) \sin \left[\varphi_{\mathbf{e}}(\mathbf{x})\right]$ using (2), whereas the monogenic approach, without the local orientation $\phi(\mathbf{x})$, actually computes the imaginary part as $a_{\mathrm{mo}}(\mathbf{x})\left|\sin \left[\varphi_{\mathrm{mo}}(\mathbf{x})\right]\right|$. Therefore, computation of the monogenic FM function involves estimation of the local orientation $\phi(\mathbf{x})$ to deduce the correct sign of the imaginary component. For instance, Larkin, Bone, and Oldfield [10] and Unser, Sage, and Ville [17] adopted the classical tensor structure orientation estimation [18].

We observe that both the local orientation $\phi(x)$ in (8) of the monogenic signal and the term $\arg \nabla \varphi_{\mathbf{e}}(\mathbf{x})$ in (5) of the pHT capture the local orientations of structures in the image $f(\mathbf{x})$. Hence, the local orientation is not required for the computation of the FM functions. Taking the derivative of the model (1) in both horizontal and vertical directions, we obtain a relationship between the derivatives of the real signal $f(\mathbf{x})$ and the FM function $\nabla \varphi_{\mathrm{mo}}(\mathbf{x})=\left[\varphi_{x}(\mathbf{x}) \varphi_{y}(\mathbf{x})\right]^{T}$ according to

$$
\begin{aligned}
& f_{x}(\mathbf{x})=a_{x}(\mathbf{x}) \cos \left[\varphi_{\mathrm{mo}}(\mathbf{x})\right]-\varphi_{x}(\mathbf{x}) a_{\mathrm{mo}}(\mathbf{x}) \sin \left[\varphi_{\mathrm{mo}}(\mathbf{x})\right] \\
& f_{y}(\mathbf{x})=a_{y}(\mathbf{x}) \cos \left[\varphi_{\mathrm{mo}}(\mathbf{x})\right]-\varphi_{y}(\mathbf{x}) a_{\mathrm{mo}}(\mathbf{x}) \sin \left[\varphi_{\mathrm{mo}}(\mathbf{x})\right]
\end{aligned}
$$


where $\nabla a_{\mathrm{mo}}(\mathbf{x})=\left[a_{x}(\mathbf{x}) a_{y}(\mathbf{x})\right]^{T}$ and $\nabla f(\mathbf{x})=\left[f_{x}(\mathbf{x}) f_{y}(\mathbf{x})\right]^{T}$. We then rearranged these derivatives to compute the magnitude of the monogenic FM functions

$$
\begin{aligned}
\left|\varphi_{x}(\mathbf{x})\right| & =\frac{\left|a_{x}(\mathbf{x}) \cos \left[\varphi_{\mathrm{mo}}(\mathbf{x})\right]-f_{x}(\mathbf{x})\right|}{\left|a_{\mathrm{mo}}(\mathbf{x}) \sin \left[\varphi_{\mathrm{mo}}(\mathbf{x})\right]\right|} \\
\left|\varphi_{y}(\mathbf{x})\right| & =\frac{\left|a_{y}(\mathbf{x}) \cos \left[\varphi_{\mathrm{mo}}(\mathbf{x})\right]-f_{y}(\mathbf{x})\right|}{\left|a_{\mathrm{mo}}(\mathbf{x}) \sin \left[\varphi_{\mathrm{mo}}(\mathbf{x})\right]\right|}
\end{aligned}
$$

where the denominator is obtained from (8) as

$$
\left|a_{\mathrm{mo}}(\mathbf{x}) \sin \left[\varphi_{\mathrm{mo}}(\mathbf{x})\right]\right|=\sqrt{f_{1}^{2}(\mathbf{x})+f_{2}^{2}(\mathbf{x})} .
$$

We calculate the orientation of the FM vector as

$$
\theta(\mathbf{x})=\arctan \left(\frac{a_{y}(\mathbf{x}) \cos \left[\varphi_{\mathrm{mo}}(\mathbf{x})\right]-f_{y}(\mathbf{x})}{a_{x}(\mathbf{x}) \cos \left[\varphi_{\mathrm{mo}}(\mathbf{x})\right]-f_{x}(\mathbf{x})}\right),
$$

where $-\pi / 2 \leq \theta(\mathbf{x}) \leq \pi / 2$. Notice that the range restriction of $\theta(\mathbf{x})$ is the result of the phase ambiguity of the model (1) where both $-\varphi_{\text {mo }}(\mathbf{x})$ and $+\varphi_{\text {mo }}(\mathbf{x})$ are valid representations because $\cos \left[\varphi_{\mathrm{mo}}(\mathbf{x})\right]$ is an even function. From (12) and (14), the FM functions are obtained according to

$$
\begin{aligned}
& \varphi_{x}(\mathbf{x})=\sqrt{\left|\varphi_{x}(\mathbf{x})\right|^{2}+\left|\varphi_{x}(\mathbf{x})\right|^{2}} \cos [\theta(\mathbf{x})] \\
& \varphi_{y}(\mathbf{x})=\sqrt{\left|\varphi_{y}(\mathbf{x})\right|^{2}+\left|\varphi_{y}(\mathbf{x})\right|^{2}} \sin [\theta(\mathbf{x})] .
\end{aligned}
$$

\section{RELATIONSHIP BETWEEN MONOGENIC AND PARTIAL HILBERT APPROACHES}

In practical applications, a multipartite signal may admit multiple orientations at a given pixel. Therefore, it is desirable to decompose the signal into individual components of different orientations so that the assumptions inherent in (1) are valid. For instance, the monogenic model has been used with multi-scale multi-orientation representations such as wavelets $[17,19]$ and the steerable pyramid [20] to analyze local signal features. Given an input signal specified by a single orientation, both the $\mathrm{pHT}$ and the monogenic approach produce the same frequency magnitude for the imaginary components $q_{\mathbf{e}}(\mathbf{x})$ and $q_{\mathrm{mo}}(\mathbf{x})$.

Assume that $s(\mathbf{x})$ is the output from one channel in a multi-scale multi-orientation realization of the steerable pyramid filterbank [21] modified as described in [22]. In particular, let $\theta_{0}$ be the orientation of the filter center frequency; the spectrum of $s(\mathbf{x})$ can be written in polar form as

$$
\widehat{s}(r, \theta)=\widehat{\xi}(r, \theta)\left[\cos \left(\theta-\theta_{0}\right)\right]^{2 \ell},
$$

where $\widehat{\xi}(r, \theta)$ is the Fourier spectrum of the original image $f(\mathbf{x})$ at a given scale and $\ell$ denotes the number of orientations per scale.

For a real signal $s(\mathbf{x})$, the Riesz transform of $s(\mathbf{x})$ produces two components $s_{1}(\mathbf{x})$ and $s_{2}(\mathbf{x})$. Similar to [10,17], we represent these two components by a complex signal $p(\mathbf{x})=s_{1}(\mathbf{x})+j s_{2}(\mathbf{x})$. Note that the complex signal $p(\mathbf{x})$ plays the role of the imaginary image in the context of the complex signal extension approach. It may be shown that the Riesz transform has unity gain:

$$
\begin{aligned}
\|\widehat{p}(\boldsymbol{\omega})\|^{2} & =\|\widehat{s}(\boldsymbol{\omega})\|^{2}\left[\frac{\omega_{x}^{2}}{\|\boldsymbol{\omega}\|^{2}}+\frac{\omega_{y}^{2}}{\|\boldsymbol{\omega}\|^{2}}\right] \\
& =\|\widehat{s}(\boldsymbol{\omega})\|^{2} .
\end{aligned}
$$

To demonstrate that the pHT also has unity gain for the specific type of signal in (17), let $g(\mathbf{x})=\mathcal{H}_{\theta_{0}}\{s(\mathbf{x})\}$, let $\mathbf{e}=$

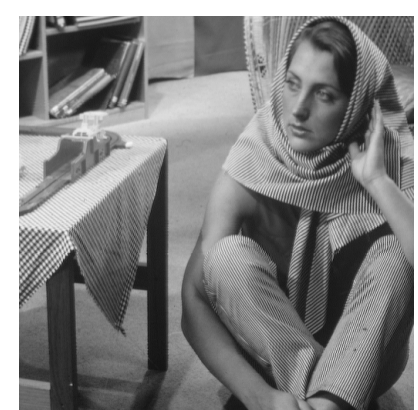

(a) Babara image

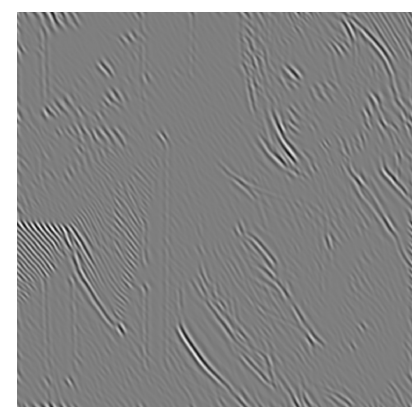

(c) Channel input image $s(\mathbf{x})$

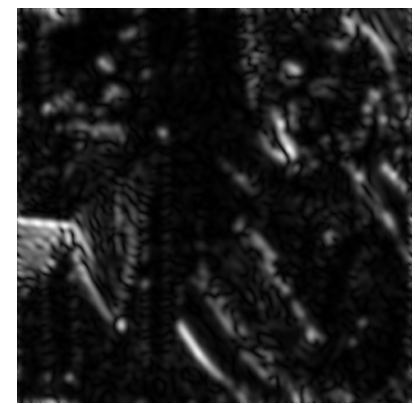

(e) Monogenic AM

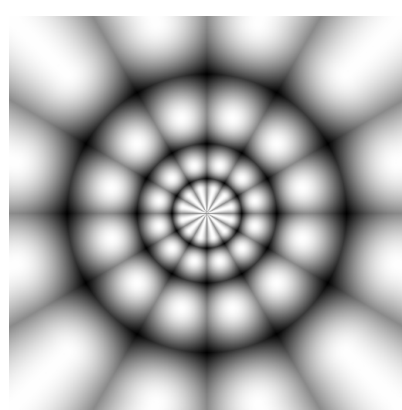

(b) Steerable pyramid spectrum

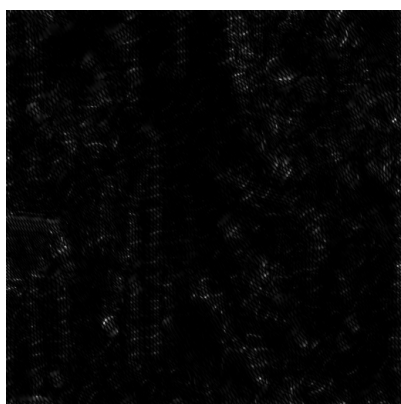

(d) Difference of (e) and (f)

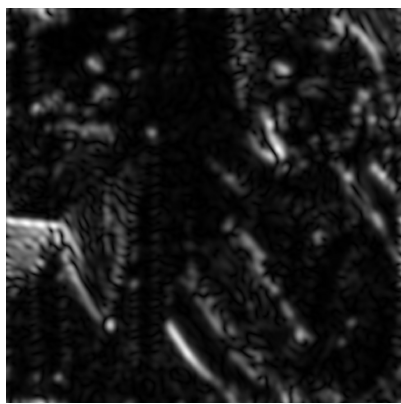

(f) $\mathrm{pHT}$ AM
Fig. 1. Computed AM using the monogenic signal and pHT.

$\left[\begin{array}{ll}\cos \left(\theta_{0}\right) & \sin \left(\theta_{0}\right)\end{array}\right]^{T}$ be the unit vector with angle $\theta_{0}$, and let $\kappa=\left[\begin{array}{ll}\cos (\theta) & \sin (\theta)\end{array}\right]^{T}$ be the polar representation of the rectangular frequency $\boldsymbol{\omega}=\left[\begin{array}{ll}\omega_{x} & \omega_{y}\end{array}\right]^{T}$. The pHT of $g(\mathbf{x})$ can be written as

$$
\begin{aligned}
\widehat{g}(r, \theta) & =-j \operatorname{sgn}\left(\kappa^{T} \mathbf{e}\right) \widehat{s}(r, \theta) \\
& =-j \operatorname{sgn}\left[\cos \left(\theta-\theta_{0}\right)\right] \widehat{s}(r, \theta) .
\end{aligned}
$$

The frequency magnitude of $g(\mathbf{x})$ is then given by

$$
\|\widehat{g}(r, \theta)\|^{2}= \begin{cases}\|\widehat{s}(r, \theta)\|^{2}, & \text { if } \cos \left(\theta-\theta_{0}\right) \neq 0, \\ 0, & \text { if } \cos \left(\theta-\theta_{0}\right)=0 .\end{cases}
$$

According to (17), $\widehat{s}(r, \theta)$ vanishes when $\cos \left(\theta-\theta_{0}\right)=0$. Hence, $\|\widehat{g}(\boldsymbol{\omega})\|^{2}=\|\widehat{s}(\boldsymbol{\omega})\|^{2}=\|\widehat{p}(\boldsymbol{\omega})\|^{2}$.

While the frequency magnitude of the imaginary signals in the two approaches are the same, the computed AM functions are not necessarily equal. Fig. 1 shows the AM computed by the two approaches. The original barbara image is given in Fig. 1(a). A spectral depiction of the modified steerable pyramid is given in Fig. 1(b). 
Fig. 1(c) shows one component of the multipartite image obtained as a steerable pyramid filterbank channel response. The absolute difference of the computed AM from the two approaches is given in Fig. 1(d), where brighter pixels denote a greater difference.

Figs. 1(e) and (f) depict the computed AM functions of the monogenic signal and the $\mathrm{pHT}$ approach respectively. In this example, the mean difference between the two AM functions is 0.022037 with reference to the range of AM functions [0, 12.3618]. While the pHT approach does not compute exactly the same AM and FM functions as those obtained by the monogenic signal, it provides an equivalent AM-FM representation.

\section{DISCUSSION AND CONCLUSION}

As noted in Sec. 2.1, the pHT kernel is not isotropic and does not have unity gain. However, for the particular signal model in (17), the pHT kernel no longer causes undesirable amplitude rippling because the spectrum $\widehat{s}(r, \theta)$ vanishes at the same places where the pHT kernel is zero. In other words, the pHT kernel can be interpreted as being effectively isotropic for signals of type (17). In addition, the $\mathrm{pHT}$ offers a simpler representation as the associated complex signal contains two components whereas the monogenic signal requires three. Because the pHT computes the imaginary image as $a(\mathbf{x}) \sin [\varphi(\mathbf{x})]$ instead of $|a(\mathbf{x}) \sin [\varphi(\mathbf{x})]|$ with the monogenic signal, we can use a simpler computation method in (5) to compute the FM function $\nabla \varphi(\mathbf{x})$ without having to estimate the local orientation $\phi(\mathbf{x})$ as is required in the pure monogenic signal approach.

In this paper, we proposed a new algorithm to compute the FM functions for the monogenic signal. We showed that the proposed algorithm is simpler than the monogenic signal model as it does not require the local orientation estimation step. In addition, we showed that in situations where a multi-scale multi-orientation signal decomposition is required to analyze a signal, both the $\mathrm{pHT}$ and the monogenic signal deliver similar AM and FM functions. However, the pHT provides simpler computations. Therefore, we advocate for use of the pHT in multi-scale multi-orientation AM-FM applications.

\section{REFERENCES}

[1] B. Boashash, "Estimating and interpreting the instantaneous frequency of a signal - Part I. Fundamentals," Proc. IEEE, vol. 80, no. 4, pp. 520-538, Apr. 1992.

[2] G. Gabor, "Theory of communication," J. Inst. Elec. Engr., vol. 93, pp. 429-457, 1946.

[3] F. Peyrin, Y. M. Zhu, and R. Goutte, "Extension of the notion of analytic signal for multidimensional signals. Application to images," in Signal Processing III: Theories and Applications, I. T. Young, et al., Ed., pp. 677-680. Elsevier Science Publishers, Amsterdam, B. V. (North-Holland), 1986.

[4] J.P. Havlicek, J.W. Havlicek, and A.C. Bovik, "The analytic image," in Proc. IEEE Int'l Conf. Image Process., Santa Barbara, CA, Oct. 26-29 1997, pp. 446-449.

[5] D. Vakman, "On the analytic signal, the Teager-Kaiser energy algorithm, and other methods for defining amplitude and frequency," IEEE Trans. Signal Proc., vol. 44, no. 4, pp. 791-797, Apr. 1996.

[6] J. P. Havlicek, D. S. Harding, and A. C. Bovik, "Multidimensional quasi-eigenfunction approximations and multicomponent AM-FM models," IEEE Trans. Image Proc., vol. 9, no. 2, pp. 227-242, Feb. 2000.
[7] S. L. Hahn, "Multidimensional complex signals with singleorthant spectra," Proc. IEEE, vol. 80, no. 8, pp. 1287-1300, Aug. 1992.

[8] T. Bülow and G. Sommer, "The hypercomplex signal - a novel extension of the analytic signal to the multidimensional case," IEEE Trans. Signal Proc., vol. 49, no. 11, pp. 2844-2852, Dec. 2001.

[9] M. Felsberg and G. Sommer, "The monogenic signal," IEEE Trans. Signal Proc., vol. 49, no. 12, pp. 3136-3144, Dec. 2001.

[10] K. G. Larkin, D. J. Bone, and M. A. Oldfield, "Natural demodulation of two-dimensional fringe patterns. I. General background on the spiral phase quadrature transform," J. Opt. Soc. Am. A, vol. 18, no. 8, pp. 1862-1870, Aug. 2001.

[11] P. Maragos and A. C. Bovik, "Image demodulation using multidimensional energy separation," J. Opt. Soc. Amer. A, vol. 12, no. 9, pp. 1867-1876, Sep. 1995.

[12] G. Girolami and D. Vakman, "Instantaneous frequency estimation and measurement: a quasi-local method," Meas. Sci. Technol., vol. 13, pp. 909-917, May 2002.

[13] V. Murray, P. Rodríguez, and M.S. Pattichis, "Multiscale AMFM demodulation and image reconstruction methods with improved accuracy," IEEE Trans. Imag. Proc., vol. 19, no. 5, pp. 1138-1152, 2010.

[14] J. L. Barron, D. J. Fleet, and S. S. Beauchemin, "Performance of optical flow techniques," Int'l Journal of Comput. Vision, vol. 12, no. 1, pp. 43-77, 1994.

[15] M.S. Pattichis and A.C. Bovik, "Analyzing image structure by multidimensional frequency modulation," IEEE Trans. Pattern Anal., Machine Intel., vol. 29, no. 5, pp. 753-766, May 2007.

[16] E. M. Stein, Singular Integrals and Differentiability Properties of Functions, Princeton University Press, Princeton, NJ, 1970.

[17] M. Unser, D. Sage, and D.V.D Ville, "Multiresolution monogenic signal analysis using the Riesz-Laplace wavelet transform," IEEE Trans. Imag. Proc., vol. 18, no. 11, pp. 24022418, Nov. 2009.

[18] H. Knutsson, "Representing local structure using tensors," in Proc. 6th Scandinavian Conf. Image Anal., Oulu, Finland, Jun. 1989, pp. 244-251.

[19] S. Olhede and G. Metikas, "The monogenic wavelet transform," IEEE Trans. Signal Proc., vol. 57, no. 9, pp. 3426-3441, Sept. 2009.

[20] S. Held, M. Storath, P. Massopust, and B. Forster, "Steerable wavelets frames based on the Riesz transform," IEEE Trans. Imag. Proc., vol. 19, no. 3, pp. 653-667, Mar. 2010.

[21] W. T. Freeman and E. H. Adelson, "The design and use of steerable filters," IEEE Trans. Pattern Anal., Machine Intel., vol. 38, no. 2, pp. 587-607, March. 1992.

[22] C. T. Nguyen and J. P. Havlicek, "AM-FM image filters," in Proc. IEEE Int'l. Conf. Image Proc., San Diego, CA, Oct. 1215, 2008, pp. 789-792. 